\title{
Increased health service use for asthma, but decreased for COPD: Northumbrian hospital episodes, 2013-2014
}

\author{
I. Shiue $\mathrm{e}^{1,2}$
}

Received: 25 August 2015 / Accepted: 7 December 2015/Published online: 15 January 2016

(C) The Author(s) 2016. This article is published with open access at Springerlink.com

\begin{abstract}
The burden of respiratory disease has persisted over the years, for both men and women. The aim of the present study was to investigate the hospital episode rates in respiratory disease and to understand whether and how the use of the health service for respiratory disease might have changed in recent years in the North-East of England. Hospital episode data covering two full calendar years (in 2013-2014) was extracted from the Northumbria Healthcare NHS Foundation Trust, which serves a population of nearly half a million. Hospital episode rates were calculated from admissions divided by annual and small area-specific population size by sex and across age groups, presented with per 100,000 person-years. The use of the health service for influenza and pneumonia, acute lower respiratory infections and chronic obstructive pulmonary disease (COPD) increased with an advancing age, except for acute upper respiratory infections and asthma. Overall, the use of the health service for common respiratory diseases has seemed to be unchanged, except for asthma. There were large increases in young adults aged 20-50 for both men and women and the very old aged
\end{abstract}

I. Shiue

ivy.shiue@northumbria.ac.uk

1 Northumbria Healthcare NHS Foundation Trust, Newcastle upon Tyne, UK

2 Department of Healthcare, Northumbria University, Newcastle upon Tyne NE1 8ST, England, UK
$90+$ in women. Of note, there were large increases in acute lower respiratory infections for both men and women aged $90+$, whereas there was also a large decrease in COPD in women aged 80-90. This is the first study to examine health service use for respiratory diseases by calculating the detailed population size as denominator. Re-diverting funding to improve population health on a yearly basis may serve the changing need in local areas.

\section{Introduction}

\section{Evidence before this study}

Respiratory disease, as an adult health condition, affects millions of people globally and is the one of the leading causes of health issues in both developed and developing countries [1]. Health service use has increased in older persons and costs millions of pounds in the UK, USA and several European countries, which could prompt considerations on long-term healthcare together with the entire socio-economic structure [2-5]. Hospital admissions have seemed to decrease in some regions, whereas in other regions primary care consultations seem to have increased, likely due to different study populations, study time periods and/or estimation methods in rates [6-28]. Continuously monitoring how people consume the health service because of various health conditions is important in assisting with individual, local and national health profiles and with the re-allocation of medical and social recourse effectively and consequently to prevent from unnecessary pain and spending. Therefore, such clinical evidence is necessary. 


\section{Knowledge gap}

Investigating admission rates and hospitalisation rates could be perceived as a direct way of understanding how many patients are admitted and hospitalised require health service utilisation. Previous research tended to estimate agestandardised rates using the population census in a certain year by accommodating a specific population structure (e.g. Europe) or by adjusting for all ages in a specific study catchment to compare across countries and/or regions. However, looking at the total age-standardised rate by using the population census in a certain year may sometimes mis- lead and misguide the re-allocation of local medical and social resources, as one national, international or global policy does not always fit all owing to different unadjusted historical contexts (i.e. biological or non-biological risk contributor profiles).

\section{Study aim}

Following this context, therefore, the aim of the present study was to investigate the age-specific hospital episode rates in common respiratory diseases by sex and across age groups using an annual and small area-
Fig. 1 Population size by sex and across age groups in Northumbria

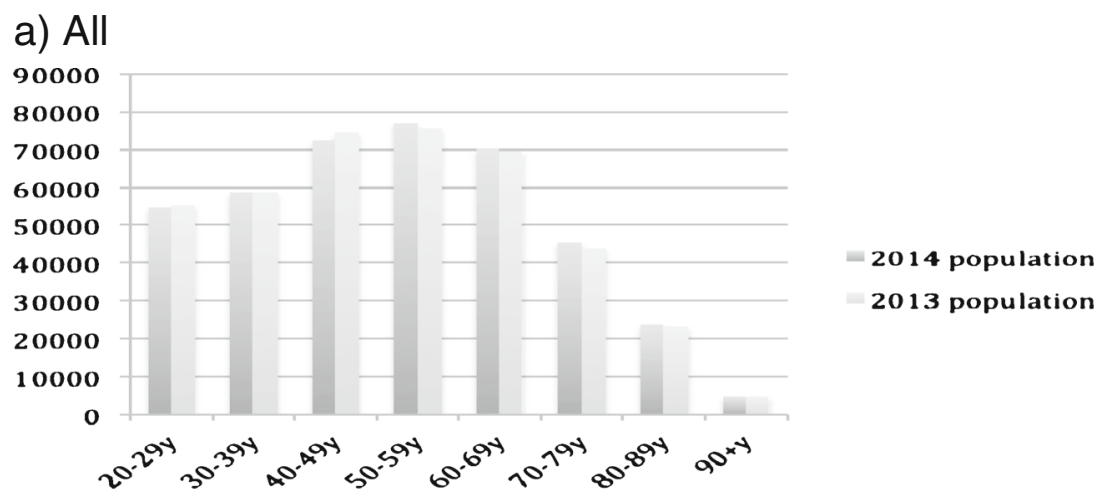

\section{b) Female}

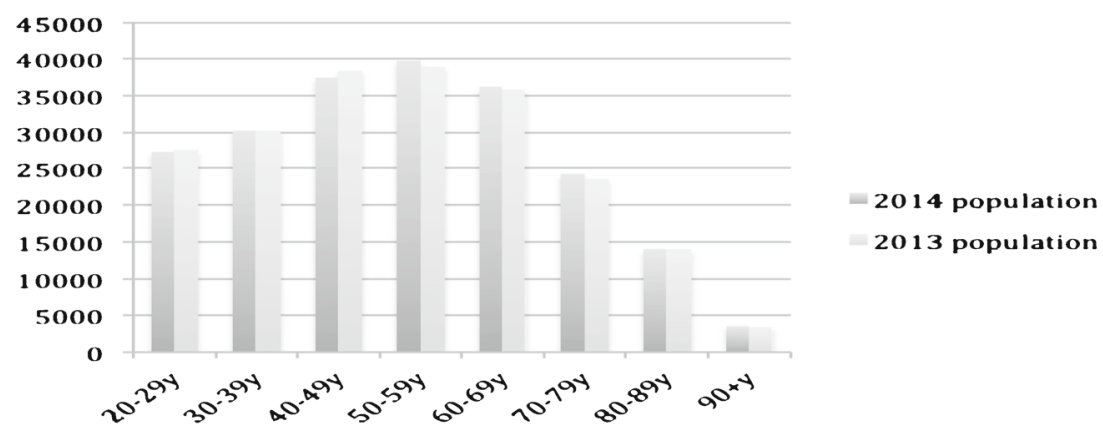

\section{c) Male}

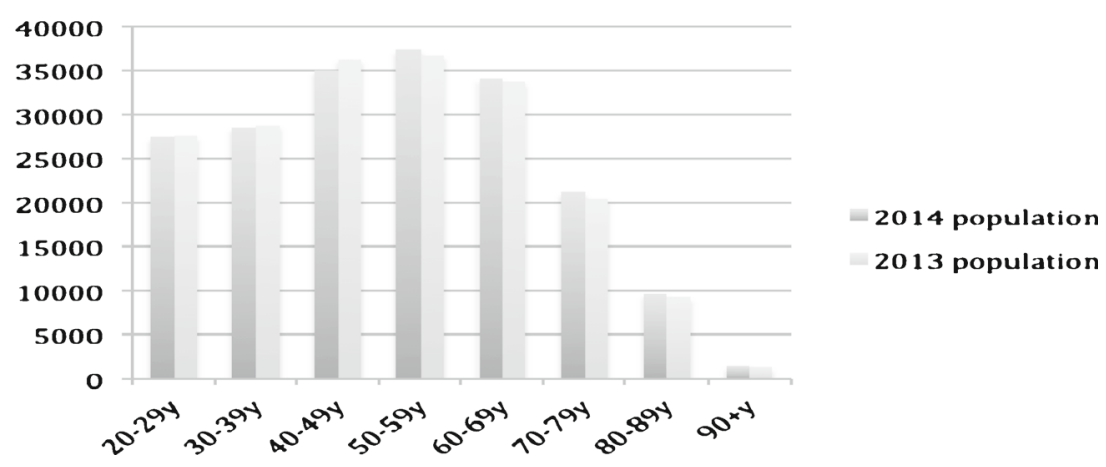


specific population size to understand and establish the monitoring on whether and how the use of the health service for respiratory diseases may have changed in recent years, if at all.

\section{Materials and methods}

\section{Study sample}

Hospital Episode Statistics (HES; more details via http:// www.hscic.gov.uk/hes) is a data warehouse containing details of all admissions, outpatient appointments and A\&E attendances at National Health Service (NHS) hospitals in England. These data are collected during a patient's time at hospital and are submitted to allow hospitals to be paid for the care they deliver. HES data are designed to enable secondary use, particularly for nonclinical purposes. Each NHS trust in England collects its own patient data, and the anonymised data are kept locally within each trust and also centrally at the national level. Northumbria Healthcare NHS Foundation Trust (more details via https://www.northumbria.nhs.uk/) covers the health service mostly for Northumberland
Fig. 2 Distribution of rates of health service use for "J00-J06: acute upper respiratory infections"

\section{a) All}

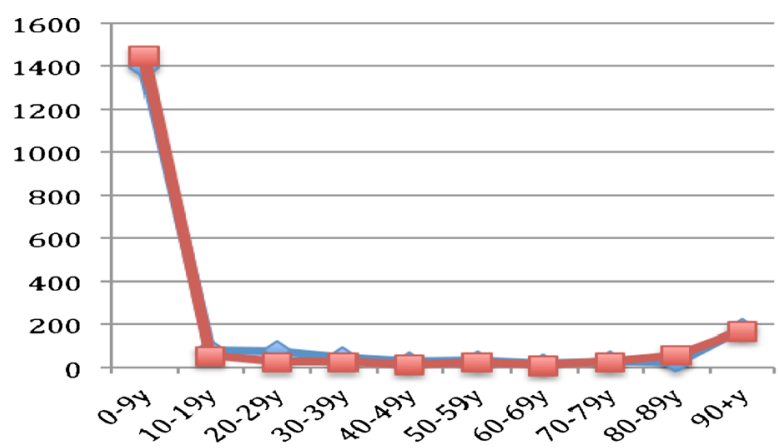

\section{b) Female}
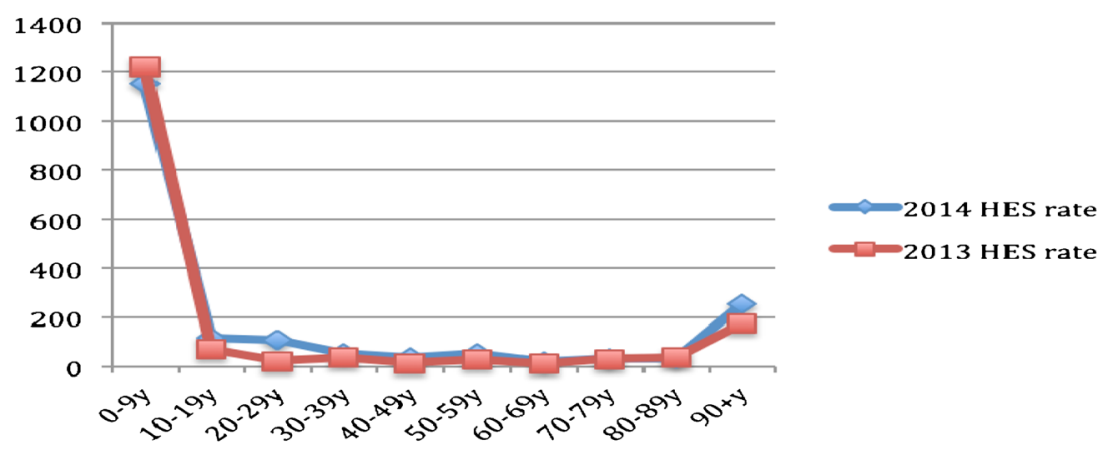

\section{c) Male}

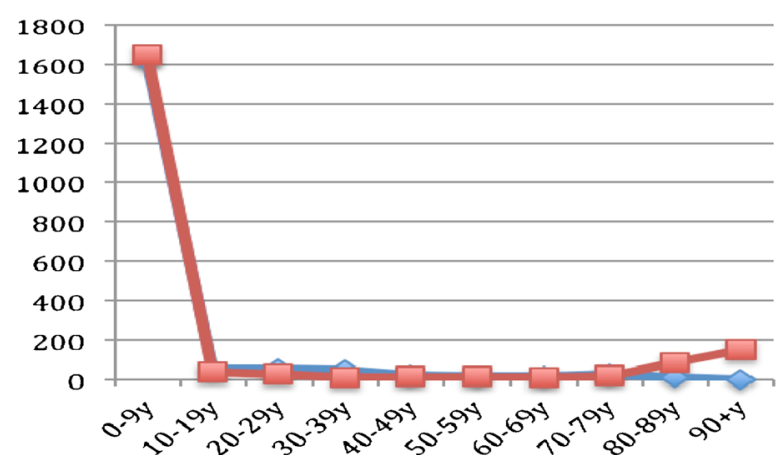

$\approx 2014$ HES rate $\square-2013$ HES rate $\approx 2014$ HES rate $\square-2013$ HES rate 
and North Tyneside, including three major hospitals (Hexham General Hospital, North Tyneside General Hospital and Wansbeck General Hospital) and other smaller community hospitals (Alnwick Infirmary, Berwick Infirmary, Blyth Community Hospital, Haltwhistle War Memorial Hospital, Rothbury Community Hospital and Sir G B Hunter Memorial
Hospital) facilitating health and social care and wellbeing for rehabilitation purposes (more details via http://www.nhs.uk/Services/Trusts/Overview/ DefaultView.aspx?id=1802) and acts as a foundation trust that has been free from central government control since 2006 (more details via https://www.northumbria.nhs.uk/ about-us/being-foundation-trust).
Fig. 3 Distribution of rates of health service use for "J09-J18: influenza and pneumonia"

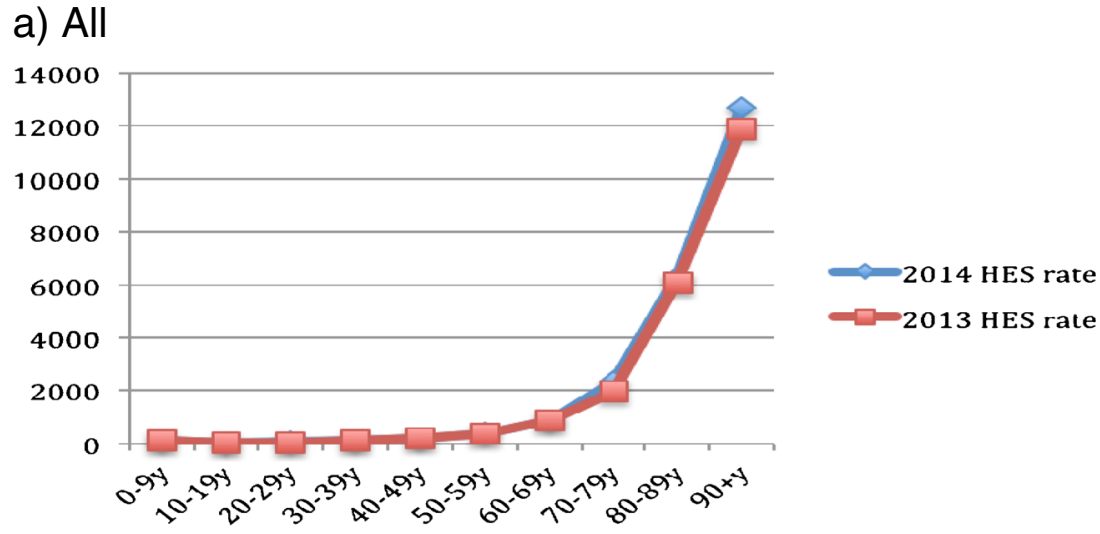

\section{b) Female}

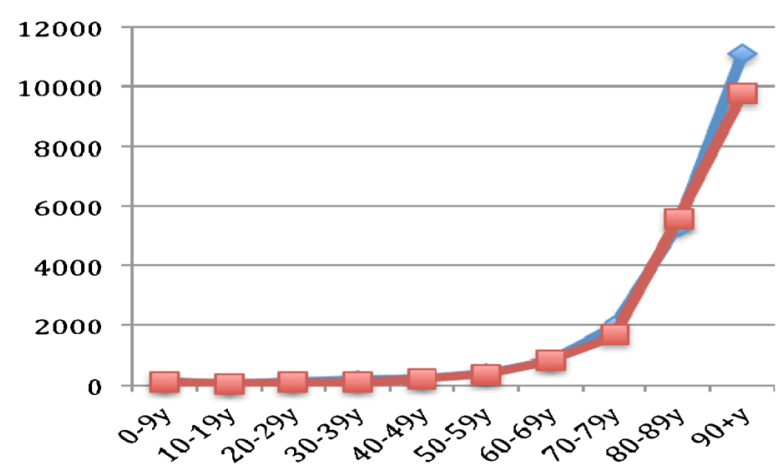

2014 HES rate $-\square 013$ HES rate

\section{c) Male}

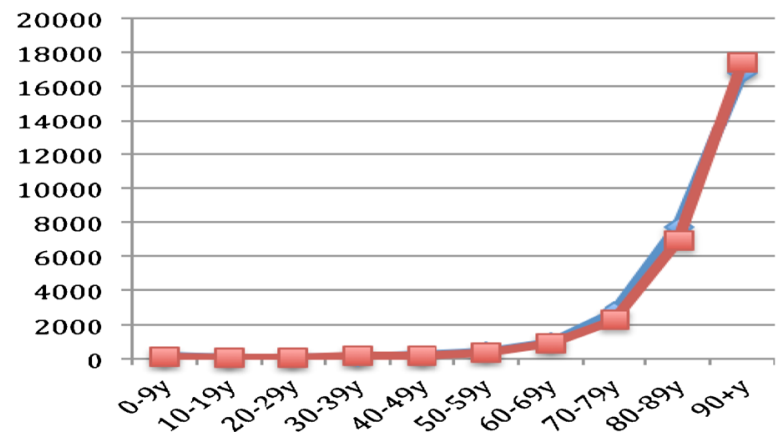

2014 HES rate $\square 2013$ HES rate 


\section{Variables and analyses}

The data from the Northumbrian Hospital Episodes used in the present study covered two full calendar years (20132014). Health service use was determined by each admission coded as J00-06 Acute upper respiratory infections, J09-18 Influenza and pneumonia, J20-J22 Acute lower respiratory infections, G44 Other chronic obstructive pulmonary disease (COPD) and J45 Asthma, based on the International
Classification of Diseases, 10th version (more details via http://apps.who.int/classifications/icd10/browse/2015/en; now re-directed to http://apps.who.int/classifications/ icd10/browse/2016/en). To estimate the usage of the health service, age-specific HES rates were calculated from admissions divided by population size for each age group, presented with per 100,000 person-years. Estimates on population size in both 2013 and 2014 were obtained from the UK Office for National
Fig. 4 Distribution of rates in health service use for "J20-J22: other acute lower respiratory infections"

\section{a) All}

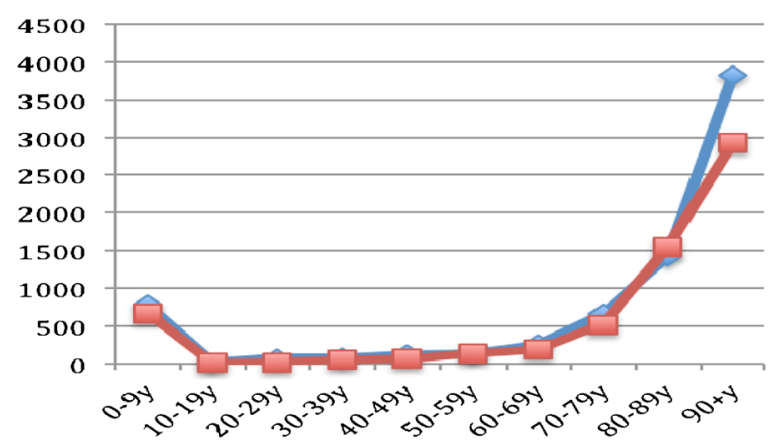

\section{b) Female}

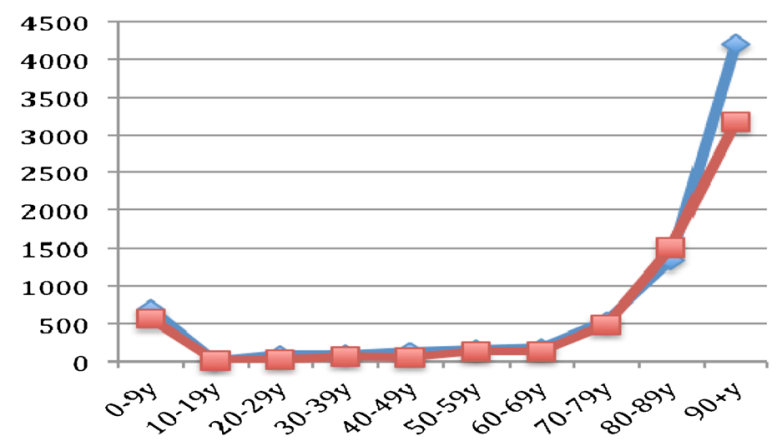

\section{c) Male}

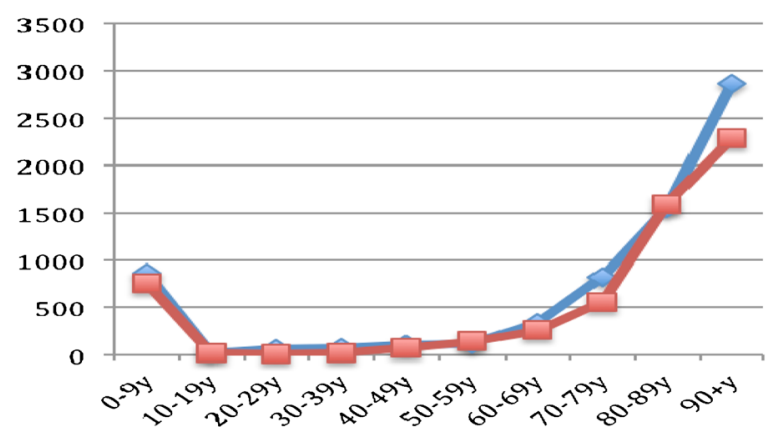

$\approx 2014$ HES rate

$-\square 2013$ HES rate

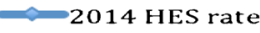

$=2013$ HES rate 
Statistics (more details via http://www.ons.gov.uk/ons/ taxonomy/index.html?nscl=Population). Statistical software STATA version 13.0 (STATA, College Station, Texas, USA; more details via http://www.stata.com/) and Microsoft Excel (more details via https://products.office. com/en-us/excel) were used to perform all the analyses and to generate graphs. As this was only a secondary data analysis with no individual identification in the present study, no further ethics approval was required.

\section{Results}

Figure 1 describes the population size by sex and across age groups in mid-2013 to mid-2014. Clearly, the population of young adults (aged 20-49) has decreased, whereas that of older adults (aged 50 and above) has increased. Figures $2-6$ show the distribution of rates of health service use for acute upper respiratory infections, influenza and pneumonia, acute
Fig. 5 Distribution of rates of health service use for "J44: COPD" (chronic obstructive pulmonary disease) a) All

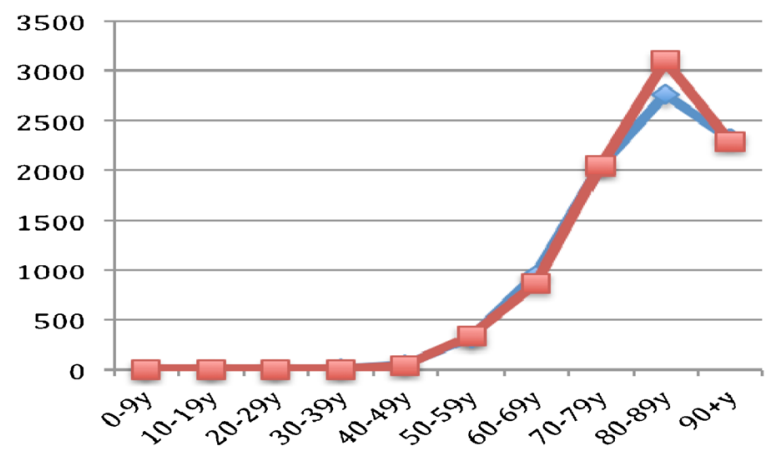

\section{b) Female}

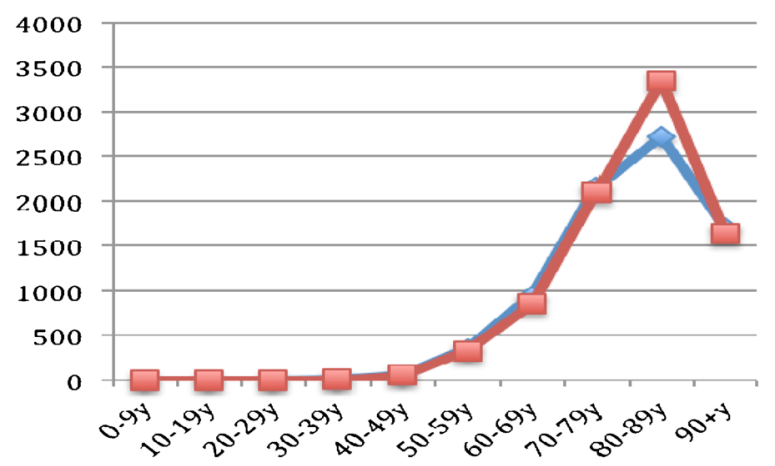

2014 HES rate $\square 2013$ HES rate

\section{c) Male}

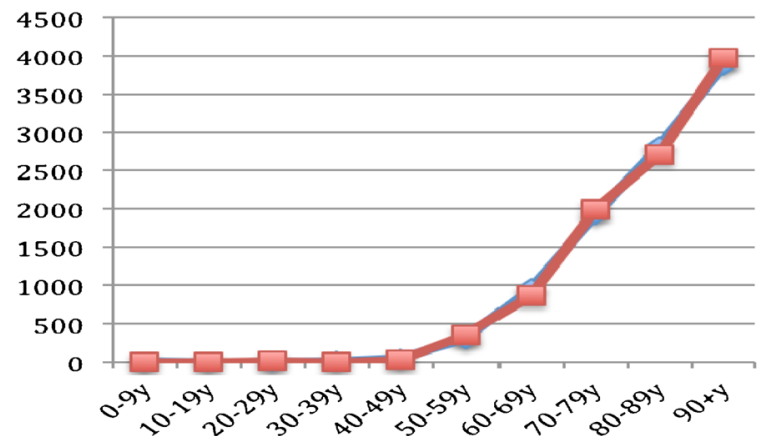

2014 HES rate $\square 2013$ HES rate 
Fig. 6 Distribution of rates of health service use for "J45:

asthma" a) All

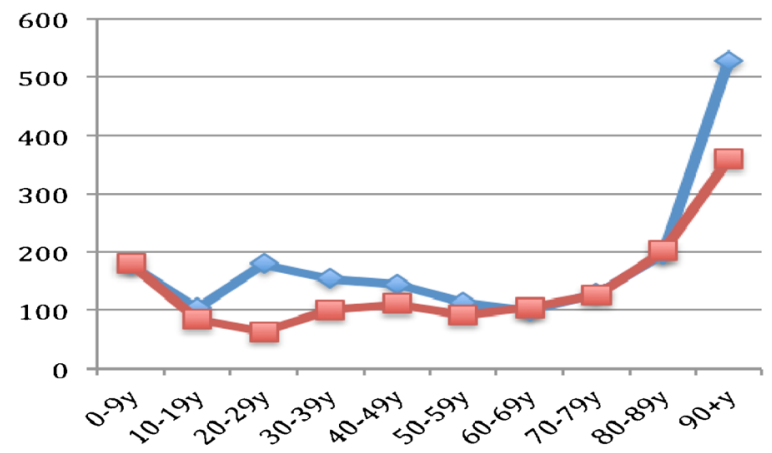

2014 HES rate

$-\square 013$ HES rate

\section{b) Female}

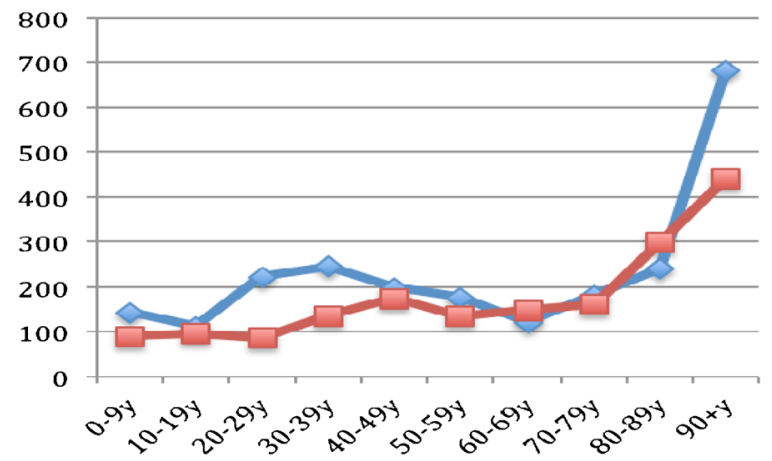

2014 HES rate $\square-2013$ HES rate

c) Male

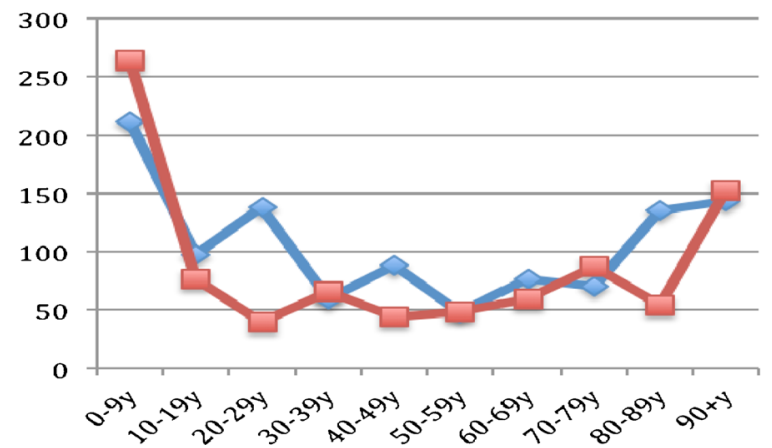

$\approx 2014$ HES rate $\square 2013$ HES rate lower respiratory infections, COPD and asthma from 2013 to 2014 by sex and age groups respectively (also see Tables 1-5). Clearly, the use of the health service for influenza and pneumonia, acute lower respiratory infections and COPD increased with an advancing age in both men and women, but not for acute upper respiratory infections and asthma. Following these 2 years, the use of the health service for common respiratory diseases has seemed to be unchanged, except for asthma. There were large increases in young adults aged 20-50 for both men and women and the very old aged 90 and above in women. Of note, there were large increases in acute lower respiratory infections for both men and women aged 90 and above; there was also a large decrease in COPD in women aged $80-90$. 
Table 1 Hospital episode statistics for "J00-J06: acute upper respiratory infections"

\begin{tabular}{|c|c|c|c|c|c|c|c|}
\hline \multicolumn{4}{|l|}{2014} & \multicolumn{4}{|l|}{2013} \\
\hline $\begin{array}{l}\text { All } \\
\text { (years) }\end{array}$ & Episode & Population & $\begin{array}{l}2014 \text { HES } \\
\text { rate }\end{array}$ & $\begin{array}{l}\text { All age groups } \\
\text { (years) }\end{array}$ & Episode & Population & $\begin{array}{l}2013 \text { HES } \\
\text { rate }\end{array}$ \\
\hline $0-9$ & 775 & 55,577 & 1394.461738 & $0-9$ & 802 & 55,550 & 1443.744374 \\
\hline $10-19$ & 47 & 55,577 & 84.567357 & $10-19$ & 30 & 56,221 & 53.36084381 \\
\hline $20-29$ & 44 & 54,879 & 80.17638805 & $20-29$ & 14 & 55,221 & 25.3526738 \\
\hline $30-39$ & 30 & 58,734 & 51.07774032 & $30-39$ & 14 & 58,955 & 23.74692562 \\
\hline $40-49$ & 21 & 72,433 & 28.99231013 & $40-49$ & 10 & 74,655 & 13.3949501 \\
\hline $50-59$ & 27 & 77,070 & 35.0330868 & $50-59$ & 16 & 75,724 & 21.12936453 \\
\hline $60-69$ & 13 & 70,296 & 18.49322863 & $60-69$ & 7 & 69,558 & 10.06354409 \\
\hline $70-79$ & 14 & 45,482 & 30.78140803 & $70-79$ & 11 & 44,044 & 24.97502498 \\
\hline $80-89$ & 6 & 23,764 & 25.2482747 & $80-89$ & 13 & 23,324 & 55.73658035 \\
\hline $90+$ & 9 & 4,919 & 182.9640171 & $90+$ & 8 & 4,716 & 169.6352841 \\
\hline Total & 164 & 40,7577 & 40.23779556 & Total & 93 & 406,197 & 22.89529465 \\
\hline \multicolumn{8}{|c|}{ Female (years) } \\
\hline $0-9$ & 309 & 26,728 & 1156.090991 & $0-9$ & 327 & 26767 & 1221.653529 \\
\hline $10-19$ & 32 & 26,938 & 118.7912985 & $10-19$ & 19 & 27247 & 69.73244761 \\
\hline $20-29$ & 30 & 27,406 & 109.4650806 & $20-29$ & 6 & 27663 & 21.68962152 \\
\hline $30-39$ & 17 & 30,170 & 56.34736493 & $30-39$ & 11 & 30200 & 36.42384106 \\
\hline $40-49$ & 14 & 37,372 & 37.4612009 & $40-49$ & 5 & 38432 & 13.00999167 \\
\hline $50-59$ & 22 & 39,723 & 55.38353095 & $50-59$ & 11 & 38943 & 28.24641142 \\
\hline $60-69$ & 7 & 36,233 & 19.31940496 & $60-69$ & 4 & 35817 & 11.16788117 \\
\hline $70-79$ & 8 & 24,226 & 33.02237266 & $70-79$ & 7 & 23546 & 29.72904103 \\
\hline $80-89$ & 5 & 14,148 & 35.3406842 & $80-89$ & 5 & 14045 & 35.5998576 \\
\hline $90+$ & 9 & 3,525 & 255.3191489 & $90+$ & 6 & 3407 & 176.1080129 \\
\hline Total & 112 & 212,803 & 52.63083697 & Total & 55 & 212053 & 25.936912 \\
\hline \multicolumn{8}{|c|}{ Male (years) } \\
\hline $0-9$ & 466 & 28,849 & 1615.30729 & $0-9$ & 475 & 28,783 & 1650.279679 \\
\hline $10-19$ & 15 & 28,609 & 52.43105317 & $10-19$ & 11 & 28,558 & 38.51810351 \\
\hline $20-29$ & 14 & 27,473 & 50.9591235 & $20-29$ & 8 & 27,558 & 29.02968285 \\
\hline $30-39$ & 13 & 28,564 & 45.51183308 & $30-39$ & 3 & 28,755 & 10.43296818 \\
\hline $40-49$ & 7 & 35,061 & 19.9652035 & $40-49$ & 5 & 36,223 & 13.80338459 \\
\hline $50-59$ & 5 & 37,347 & 13.38795619 & $50-59$ & 5 & 36,781 & 13.59397515 \\
\hline $60-69$ & 6 & 34,063 & 17.61442034 & $60-69$ & 3 & 33,741 & 8.891259892 \\
\hline $70-79$ & 6 & 21,256 & 28.22732405 & $70-79$ & 4 & 20,498 & 19.51409894 \\
\hline $80-89$ & 1 & 9,616 & 10.39933444 & $80-89$ & 8 & 9,279 & 86.21618709 \\
\hline $90+$ & 0 & 1,394 & 0 & $90+$ & 2 & 1,309 & 152.7883881 \\
\hline Total & 52 & 194,774 & 26.69760851 & Total & 38 & 194,144 & 19.57310038 \\
\hline
\end{tabular}

\section{Discussion}

Methodologically, there are a number of ways of examining hospital admissions, i.e. the use of the health service, in the population. To be specific, we could look historically at the trends by day of the week, by month, by season or by year. We could also examine geographically by hospital, by city, by region or by country. Mathematically, we could estimate by number, by rate or by standardisation. Politically, we could assess by practice, by policy or by reform. For example, respiratory admissions declined accompanying an increase in smoke-free areas or with the introduction of immunisation [29-33]. Understanding the use of the health service in the bigger picture is critical for health service providers and policy makers to effectively re-allocate medical and social resources (from prevention to rehabilitation) respectively. The targeted at-risk population may shift following the change in investment in health and nursing 
Table 2 Hospital episode statistics for "J09-J18: influenza and pneumonia"

\begin{tabular}{|c|c|c|c|c|c|c|c|}
\hline \multicolumn{4}{|l|}{2014} & \multicolumn{4}{|l|}{2013} \\
\hline $\begin{array}{l}\text { All } \\
\text { (years) }\end{array}$ & Episode & Population & $\begin{array}{l}2014 \text { HES } \\
\text { rate }\end{array}$ & $\begin{array}{l}\text { All age groups } \\
\text { (years) }\end{array}$ & Episode & Population & $\begin{array}{l}2013 \text { HES } \\
\text { rate }\end{array}$ \\
\hline $0-9$ & 67 & 55,577 & 120.5534664 & $0-9$ & 66 & 55,550 & 118.8118812 \\
\hline $10-19$ & 26 & 55,577 & 46.78194217 & $10-19$ & 16 & 56,221 & 28.4591167 \\
\hline $20-29$ & 41 & 54,879 & 74.70981614 & $20-29$ & 31 & 55,221 & 56.13806342 \\
\hline $30-39$ & 73 & 58,734 & 124.2891681 & $30-39$ & 75 & 58,955 & 127.215673 \\
\hline $40-49$ & 147 & 72,433 & 202.9461709 & $40-49$ & 147 & 74,655 & 196.9057665 \\
\hline $50-59$ & 312 & 77,070 & 404.8267808 & $50-59$ & 272 & 75,724 & 359.1991971 \\
\hline $60-69$ & 620 & 70,296 & 881.9847502 & $60-69$ & 600 & 69,558 & 862.5894937 \\
\hline $70-79$ & 1,069 & 45,482 & $2,350.38037$ & $70-79$ & 868 & 44,044 & $1,970.756516$ \\
\hline $80-89$ & 1,494 & 23,764 & $6,286.820401$ & $80-89$ & 1,420 & 23,324 & $6,088.149546$ \\
\hline $90+$ & 625 & 4,919 & $12,705.83452$ & $90+$ & 561 & 4,716 & $11,895.6743$ \\
\hline Total & 4,474 & 407,577 & $1,097.706691$ & Total & 4,056 & 406, 197 & 998.5302698 \\
\hline \multicolumn{8}{|c|}{ Female (years) } \\
\hline $0-9$ & 28 & 26,728 & 104.7590542 & $0-9$ & 27 & 26,767 & 100.8704748 \\
\hline $10-19$ & 10 & 26,938 & 37.12228079 & $10-19$ & 9 & 27,247 & 33.03115939 \\
\hline $20-29$ & 25 & 27,406 & 91.22090053 & $20-29$ & 24 & 27,663 & 86.75848606 \\
\hline $30-39$ & 46 & 30,170 & 152.4693404 & $30-39$ & 29 & 30,200 & 96.02649007 \\
\hline $40-49$ & 76 & 37,372 & 203.3608049 & $40-49$ & 80 & 38,432 & 208.1598668 \\
\hline $50-59$ & 156 & 39,723 & 392.7195831 & $50-59$ & 141 & 38,943 & 362.0676373 \\
\hline $60-69$ & 300 & 36,233 & 827.9744984 & $60-69$ & 300 & 35,817 & 837.591088 \\
\hline $70-79$ & 482 & 24,226 & $1,989.597953$ & $70-79$ & 398 & 23,546 & $1,690.308333$ \\
\hline $80-89$ & 750 & 14,148 & $5,301.102629$ & $80-89$ & 780 & 14,045 & $5,553.577786$ \\
\hline $90+$ & 391 & 3,525 & $11,092.19858$ & $90+$ & 333 & 3,407 & $9,773.994717$ \\
\hline Total & 2,264 & 212,803 & $1,063.894776$ & Total & 2,121 & 212,053 & $1,000.221643$ \\
\hline \multicolumn{8}{|c|}{ Male (years) } \\
\hline $0-9$ & 39 & 28,849 & 135.1866616 & $0-9$ & 39 & 28,783 & 135.4966473 \\
\hline $10-19$ & 16 & 28,609 & 55.92645671 & $10-19$ & 7 & 28,558 & 24.51152041 \\
\hline $20-29$ & 16 & 27,473 & 58.23899829 & $20-29$ & 7 & 27,558 & 25.40097249 \\
\hline $30-39$ & 27 & 28,564 & 94.52457639 & $30-39$ & 46 & 28,755 & 159.9721788 \\
\hline $40-49$ & 71 & 35,061 & 202.504207 & $40-49$ & 67 & 36,223 & 184.9653535 \\
\hline $50-59$ & 156 & 37,347 & 417.7042333 & $50-59$ & 131 & 36,781 & 356.1621489 \\
\hline $60-69$ & 320 & 34,063 & 939.4357514 & $60-69$ & 300 & 33,741 & 889.1259892 \\
\hline $70-79$ & 587 & 21,256 & $2,761.573203$ & $70-79$ & 470 & 20,498 & $2,292.906625$ \\
\hline $80-89$ & 744 & 9,616 & $7,737.104825$ & $80-89$ & 640 & 9,279 & $6,897.294967$ \\
\hline $90+$ & 234 & 1,394 & $16,786.22669$ & $90+$ & 228 & 1,309 & $17,417.87624$ \\
\hline Total & 2,210 & 194,774 & $1,134.648362$ & total & 1,935 & 194,144 & 996.6828746 \\
\hline
\end{tabular}

programs and the subsequent risk contributor profile (biologically or non-biologically). Therefore, the performance review of such ought to be documented regularly, preferably annually.

\section{Strengths and limitations}

The present study has a few strengths. First, the data are from recent years. Therefore, the results provide information on recent health policy use. Second, the study period covers full calendar years. In addition, the population size was estimated on a yearly basis. Therefore, selection bias could be avoided in the presentation of trends and the estimation of rates could be more accurate than using the population census from a single year. However, mis-classification may not be completely avoidable [34, 35]. Third, this is the first HES study looking at the use of the health service in 
Table 3 Hospital episode statistics for "J20-J22: other acute lower respiratory infections"

\begin{tabular}{|c|c|c|c|c|c|c|c|}
\hline \multicolumn{4}{|l|}{2014} & \multicolumn{4}{|l|}{2013} \\
\hline $\begin{array}{l}\text { All } \\
\text { (years) }\end{array}$ & Episode & Population & $\begin{array}{l}2014 \text { HES } \\
\text { rate }\end{array}$ & $\begin{array}{l}\text { All age groups } \\
\text { (years) }\end{array}$ & Episode & Population & $\begin{array}{l}2013 \text { HES } \\
\text { rate }\end{array}$ \\
\hline $0-9$ & 436 & 55,577 & 784.4971841 & $0-9$ & 372 & 55,550 & 669.6669667 \\
\hline $10-19$ & 10 & 55,577 & 17.99305468 & $10-19$ & 9 & 56,221 & 16.00825314 \\
\hline $20-29$ & 40 & 54,879 & 72.8876255 & $20-29$ & 10 & 55,221 & 18.10905272 \\
\hline $30-39$ & 48 & 58,734 & 81.72438451 & $30-39$ & 28 & 58,955 & 47.49385124 \\
\hline $40-49$ & 83 & 72,433 & 114.5886543 & $40-49$ & 49 & 74,655 & 65.63525551 \\
\hline $50-59$ & 105 & 77,070 & 136.239782 & $50-59$ & 102 & 75,724 & 134.6996989 \\
\hline $60-69$ & 180 & 70,296 & 256.0600888 & $60-69$ & 134 & 69,558 & 192.6449869 \\
\hline 70-79 & 304 & 45,482 & 668.3962886 & $70-79$ & 229 & 44,044 & 519.9346108 \\
\hline $80-89$ & 339 & 23,764 & $1,426.527521$ & $80-89$ & 359 & 23,324 & $1,539.187103$ \\
\hline $90+$ & 188 & 4,919 & $3,821.915023$ & $90+$ & 138 & 4,716 & $2,926.208651$ \\
\hline Total & 1,733 & 407,577 & 425.1957299 & Total & 1,430 & 406,197 & 352.0459285 \\
\hline \multicolumn{8}{|c|}{ Female (years) } \\
\hline $0-9$ & 186 & 26,728 & 695.8994313 & $0-9$ & 153 & 26,767 & 571.5993574 \\
\hline $10-19$ & 3 & 26,938 & 11.13668424 & $10-19$ & 3 & 27,247 & 11.01038646 \\
\hline $20-29$ & 23 & 27,406 & 83.92322849 & $20-29$ & 8 & 27,663 & 28.91949535 \\
\hline $30-39$ & 27 & 30,170 & 89.49287372 & $30-39$ & 21 & 30,200 & 69.53642384 \\
\hline $40-49$ & 46 & 37,372 & 123.086803 & $40-49$ & 22 & 38,432 & 57.24396336 \\
\hline $50-59$ & 63 & 39,723 & 158.5982932 & $50-59$ & 50 & 38,943 & 128.3927792 \\
\hline $60-69$ & 65 & 36,233 & 179.3944747 & $60-69$ & 46 & 35,817 & 128.4306335 \\
\hline 70-79 & 129 & 24,226 & 532.4857591 & 70-79 & 114 & 23,546 & 484.1586681 \\
\hline $80-89$ & 190 & 14,148 & $1,342.945999$ & $80-89$ & 211 & 14,045 & $1,502.313991$ \\
\hline $90+$ & 148 & 3,525 & $4,198.58156$ & $90+$ & 108 & 3,407 & $3,169.944232$ \\
\hline Total & 880 & 212,803 & 413.5280048 & Total & 736 & 212,053 & 347.0830406 \\
\hline \multicolumn{8}{|c|}{ Male (years) } \\
\hline $0-9$ & 250 & 28,849 & 866.581164 & $0-9$ & 219 & 28,783 & 760.8657888 \\
\hline 10-19 & 7 & 28,609 & 24.46782481 & $10-19$ & 6 & 28,558 & 21.00987464 \\
\hline $20-29$ & 17 & 27,473 & 61.87893568 & $20-29$ & 2 & 27,558 & 7.257420713 \\
\hline $30-39$ & 21 & 28,564 & 73.51911497 & $30-39$ & 7 & 28,755 & 24.34359242 \\
\hline $40-49$ & 37 & 35,061 & 105.5303614 & $40-49$ & 27 & 36,223 & 74.53827679 \\
\hline $50-59$ & 42 & 37,347 & 112.458832 & $50-59$ & 52 & 36,781 & 141.3773416 \\
\hline $60-69$ & 115 & 34,063 & 337.6097232 & $60-69$ & 88 & 33,741 & 260.8102902 \\
\hline 70-79 & 175 & 21,256 & 823.2969514 & 70-79 & 115 & 20,498 & 561.0303444 \\
\hline $80-89$ & 149 & 9,616 & $1,549.500832$ & $80-89$ & 148 & 9,279 & $1,594.999461$ \\
\hline $90+$ & 40 & 1,394 & $2,869.440459$ & $90+$ & 30 & 1,309 & $2,291.825821$ \\
\hline Total & 853 & 194,774 & 437.9434627 & Total & 694 & 194,144 & 357.4666227 \\
\hline
\end{tabular}

respiratory disease from the Northumbria area, which is free from central governmental control. However, there are also a few limitations that cannot be ignored. First, it was not possible to link with population surveys to understand patient risk contributor profiles, whether biological or non-biological. However, the entire study focus was to investigate if and how different age groups could present any change in health service use in recent years. Second, only two genders were identified. In other words, transgender was not properly coded.
Therefore, no results on transgender people could be obtained (more details via http://www.ons.gov.uk/ons/ about-ons/business-transparency/freedom-of-information/ what-can-i-request/previous-foi-requests/health-andsocial-care/transgender-population-figures/index.html). Third, some coding errors might not be $100 \%$ avoidable, which would affect the estimates. Taken together, future studies retaining the strengths and overcoming the limitations mentioned above to continuously monitor and document such clinical 
Table 4 Hospital episode statistics for "J44: COPD" (chronic obstructive pulmonary disease)

\begin{tabular}{|c|c|c|c|c|c|c|c|}
\hline \multicolumn{4}{|l|}{2014} & \multicolumn{4}{|l|}{2013} \\
\hline All (years) & Episode & Population & 2014 HES rate & All & Episode & Population & 2013 HES rate \\
\hline $0-9$ & 1 & 55,577 & 1.799305468 & $0-9$ & 0 & 55,550 & 0 \\
\hline $10-19$ & 0 & 55,577 & 0 & $10-19$ & 0 & 56,221 & 0 \\
\hline $20-29$ & 0 & 54,879 & 0 & $20-29$ & 2 & 55,221 & 3.621810543 \\
\hline $30-39$ & 6 & 58,734 & 10.21554806 & $30-39$ & 1 & 58,955 & 1.696208973 \\
\hline $40-49$ & 35 & 72,433 & 48.32051689 & $40-49$ & 30 & 74,655 & 40.18485031 \\
\hline $50-59$ & 245 & 77,070 & 317.8928247 & $50-59$ & 255 & 75,724 & 336.7492473 \\
\hline $60-69$ & 670 & 70,296 & 953.1125526 & $60-69$ & 598 & 69,558 & 859.7141953 \\
\hline $70-79$ & 930 & 45,482 & $2,044.764962$ & $70-79$ & 902 & 44,044 & $2,047.952048$ \\
\hline $80-89$ & 656 & 23,764 & $2,760.478034$ & $80-89$ & 722 & 23,324 & $3,095.523924$ \\
\hline $90+$ & 114 & 4,919 & $2,317.544216$ & $90+$ & 108 & 4,716 & $2,290.076336$ \\
\hline Total & 2,657 & 407,577 & 651.9013585 & Total & 2,618 & 406,197 & 644.5148536 \\
\hline \multicolumn{8}{|c|}{ Female (years) } \\
\hline $0-9$ & 0 & 26,728 & 0 & $0-9$ & 0 & 26,767 & 0 \\
\hline $10-19$ & 0 & 26,938 & 0 & $10-19$ & 0 & 27,247 & 0 \\
\hline $20-29$ & 0 & 27,406 & 0 & $20-29$ & 0 & 27,663 & 0 \\
\hline $30-39$ & 4 & 30,170 & 13.25820351 & $30-39$ & 2 & 30,200 & 6.622516556 \\
\hline $40-49$ & 20 & 37,372 & 53.51600128 & $40-49$ & 21 & 38,432 & 54.64196503 \\
\hline $50-59$ & 136 & 39,723 & 342.3709186 & $50-59$ & 127 & 38,943 & 326.1176591 \\
\hline $60-69$ & 342 & 36,233 & 943.8909282 & $60-69$ & 304 & 35,817 & 848.7589692 \\
\hline $70-79$ & 521 & 24,226 & $2,150.582019$ & $70-79$ & 493 & 23,546 & $2,093.773889$ \\
\hline $80-89$ & 385 & 14,148 & $2,721.232683$ & $80-89$ & 471 & 14,045 & $3,353.506586$ \\
\hline $90+$ & 60 & 3,525 & $1,702.12766$ & $90+$ & 56 & 3,407 & $1,643.674787$ \\
\hline Total & 1,468 & 212,803 & 689.8398989 & Total & 1,474 & 212,053 & 695.1092416 \\
\hline \multicolumn{8}{|c|}{ Male (years) } \\
\hline $0-9$ & 1 & 28,849 & 3.466324656 & $0-9$ & 0 & 28,783 & 0 \\
\hline $10-19$ & 0 & 28,609 & 0 & $10-19$ & 0 & 28,558 & 0 \\
\hline $20-29$ & 0 & 27,473 & 0 & $20-29$ & 2 & 27,558 & 7.257420713 \\
\hline $30-39$ & 2 & 28,564 & 7.001820473 & $30-39$ & 0 & 28,755 & 0 \\
\hline $40-49$ & 15 & 35,061 & 42.78257893 & $40-49$ & 9 & 36,223 & 24.84609226 \\
\hline $50-59$ & 109 & 37,347 & 291.857445 & $50-59$ & 128 & 36,781 & 348.0057638 \\
\hline $60-69$ & 328 & 34,063 & 962.9216452 & $60-69$ & 294 & 33,741 & 871.3434694 \\
\hline $70-79$ & 409 & 21,256 & 1924.162589 & $70-79$ & 409 & 20,498 & $1,995.316616$ \\
\hline $80-89$ & 271 & 9,616 & 2818.219634 & $80-89$ & 251 & 9,279 & $2,705.03287$ \\
\hline $90+$ & 54 & 1,394 & 3873.74462 & $90+$ & 52 & 1,309 & $3,972.49809$ \\
\hline Total & 1,189 & 194,774 & 610.4510869 & Total & 1,145 & 194,144 & 589.7684193 \\
\hline
\end{tabular}

evidence from the local setting to the national setting would be recommended.

\section{Research, practice and policy implications}

From 2013 to 2014, there has been unchanged use of health service utilisation with regard to common respiratory diseases, except for asthma. Respiratory disease is a common condition that has a large and negative impact on quality of life and life expectancy, with high financial costs. To direct future research, local health policy and guidelines could benefit from annual clinical records on health service use for respiratory diseases. From the practice and policy perspectives, reorganising and re-diverting funding to improve population health on a yearly basis, including improving the role of health and nursing professionals in reducing the burden of 
Table 5 Hospital episode statistics for "J45: asthma"

\begin{tabular}{|c|c|c|c|c|c|c|c|}
\hline \multicolumn{4}{|l|}{2014} & \multicolumn{4}{|l|}{2013} \\
\hline All (years) & Episode & Population & 2014 HES rate & All & Episode & Population & 2013 HES rate \\
\hline $0-9$ & 99 & 55,577 & 178.1312413 & $0-9$ & 100 & 55,550 & 180.0180018 \\
\hline $10-19$ & 58 & 55,577 & 104.3597171 & $10-19$ & 48 & 56,221 & 85.3773501 \\
\hline $20-29$ & 99 & 54,879 & 180.3968731 & $20-29$ & 35 & 55,221 & 63.3816845 \\
\hline $30-39$ & 91 & 58,734 & 154.9358123 & $30-39$ & 60 & 58,955 & 101.7725384 \\
\hline $40-49$ & 105 & 72,433 & 144.9615507 & $40-49$ & 83 & 74,655 & 111.1780859 \\
\hline $50-59$ & 88 & 77,070 & 114.1819125 & $50-59$ & 70 & 75,724 & 92.44096984 \\
\hline $60-69$ & 70 & 70,296 & 99.57892341 & $60-69$ & 73 & 69,558 & 104.9483884 \\
\hline $70-79$ & 59 & 45,482 & 129.7216481 & $70-79$ & 56 & 44,044 & 127.1455817 \\
\hline $80-89$ & 47 & 23,764 & 197.7781518 & $80-89$ & 47 & 23,324 & 201.5091751 \\
\hline $90+$ & 26 & 4,919 & 528.562716 & $90+$ & 17 & 4,716 & 360.4749788 \\
\hline Total & 742 & 407,577 & 182.0514897 & Total & 589 & 406,197 & 145.0035328 \\
\hline \multicolumn{8}{|c|}{ Female (years) } \\
\hline $0-9$ & 38 & 26,728 & 142.1730021 & $0-9$ & 24 & 26,767 & 89.6626443 \\
\hline $10-19$ & 30 & 26,938 & 111.3668424 & $10-19$ & 26 & 27,247 & 95.42334936 \\
\hline $20-29$ & 61 & 27,406 & 222.5789973 & $20-29$ & 24 & 27,663 & 86.75848606 \\
\hline $30-39$ & 74 & 30,170 & 245.276765 & $30-39$ & 41 & 30,200 & 135.7615894 \\
\hline $40-49$ & 74 & 37,372 & 198.0092048 & $40-49$ & 67 & 38,432 & 174.3338884 \\
\hline $50-59$ & 70 & 39,723 & 176.2203258 & $50-59$ & 52 & 38,943 & 133.5284904 \\
\hline $60-69$ & 44 & 36,233 & 121.4362598 & $60-69$ & 53 & 35,817 & 147.9744256 \\
\hline $70-79$ & 44 & 24,226 & 181.6230496 & $70-79$ & 38 & 23,546 & 161.3862227 \\
\hline $80-89$ & 34 & 14,148 & 240.3166525 & $80-89$ & 42 & 14,045 & 299.0388038 \\
\hline $90+$ & 24 & 3,525 & 680.8510638 & $90+$ & 15 & 3,407 & 440.2700323 \\
\hline Total & 493 & 212,803 & 231.6696663 & Total & 382 & 212,053 & 180.1436433 \\
\hline \multicolumn{8}{|c|}{ Male (years) } \\
\hline $0-9$ & 61 & 28,849 & 211.445804 & $0-9$ & 76 & 28,783 & 264.0447486 \\
\hline $10-19$ & 28 & 28,609 & 97.87129924 & $10-19$ & 22 & 28,558 & 77.03620702 \\
\hline $20-29$ & 38 & 27,473 & 138.3176209 & $20-29$ & 11 & 27,558 & 39.91581392 \\
\hline $30-39$ & 17 & 28,564 & 59.51547402 & $30-39$ & 19 & 28,755 & 66.07546514 \\
\hline $40-49$ & 31 & 35,061 & 88.4173298 & $40-49$ & 16 & 36,223 & 44.17083069 \\
\hline $50-59$ & 18 & 37,347 & 48.1966423 & $50-59$ & 18 & 36,781 & 48.93831054 \\
\hline $60-69$ & 26 & 34,063 & 76.3291548 & $60-69$ & 20 & 33,741 & 59.27506594 \\
\hline $70-79$ & 15 & 21,256 & 70.56831012 & $70-79$ & 18 & 20,498 & 87.81344521 \\
\hline $80-89$ & 13 & 9,616 & 135.1913478 & $80-89$ & 5 & 9,279 & 53.88511693 \\
\hline $90+$ & 2 & 1,394 & 143.472023 & $90+$ & 2 & 1,309 & 152.7883881 \\
\hline Total & 249 & 194,774 & 127.8404715 & Total & 207 & 194,144 & 106.6218889 \\
\hline
\end{tabular}

rehabilitation and raising public awareness, attitude and knowledge may serve the changing need in local areas.

\section{Compliance with ethical standards}

Conflicts of interest None.
Open Access This article is distributed under the terms of the Creative Commons Attribution 4.0 International License (http:// creativecommons.org/licenses/by/4.0/), which permits unrestricted use, distribution, and reproduction in any medium, provided you give appropriate credit to the original author(s) and the source, provide a link to the Creative Commons license, and indicate if changes were made. 


\section{References}

1. Global Burden of Disease Study 2013 Collaborators (2015) Global, regional, and national incidence, prevalence, and years lived with disability for 301 acute and chronic diseases and injuries in 188 countries, 1990-2013: a systematic analysis for the Global Burden of Disease Study 2013. Lancet. doi:10.1016/S0140-6736(15)60692-4

2. Oppong R, Coast J, Hood K, Nuttall J, Smith RD, Butler CC; GRACE-01 Study Team (2011) Resource use and costs of treating acute cough/lower respiratory tract infections in 13 European countries: results and challenges. Eur J Health Econ 12(4):319-329

3. Khakban A, Sin DD, FitzGerald JM, Ng R, Zafari Z, McManus B, Hollander Z, Marra CA, Sadatsafavi M (2015) 10-year trends in direct costs of COPD: a population based study. Chest 148(3):640-646

4. Stewart DL, Romero JR, Buysman EK, Fernandes AW, Mahadevia PJ (2009) Total healthcare costs in the US for preterm infants with respiratory syncytial virus lower respiratory infection in the first year of life requiring medical attention. Curr Med Res Opin 25(11):2795-2804

5. Monte SV, Paolini NM, Slazak EM, Schentag JJ, Paladino JA (2008) Costs of treating lower respiratory tract infections. Am J Manag Care 14(4):190-196

6. Singleton RJ, Holman RC, Folkema AM, Wenger JD, Steiner CA, Redd JT (2012) Trends in lower respiratory tract infection hospitalizations among American Indian/Alaska Native children and the general US child population. J Pediatr 161(2):296-302.e2

7. Moore H, Burgner D, Carville K, Jacoby P, Richmond P, Lehmann D (2007) Diverging trends for lower respiratory infections in nonAboriginal and Aboriginal children. J Paediatr Child Health 43(6): $451-457$

8. Fleming D, Harcourt S, Smith G (2003) Influenza and adult hospital admissions for respiratory conditions in England 1989-2001. Commun Dis Public Health 6(3):231-237

9. Aldaz P, Loayssa JR, Apezteguía J, Oscariz M, Dronda MJ, Sagredo M, Castilla J (2011) Increased primary care consultations for upper respiratory tract infections and for fever coinciding with a wave of influenza. Rev Esp Salud Publica 85(1):113-120

10. Hatziagorou E, Kirvassilis F, Saraphidou S, Katsara M, Valeri R, Emporiadou M, Magnisali C, Tsanakas J (2009) Acute respiratory admissions in Thessaloniki, Greece: 14-year follow-up. Hippokratia 13(4):242-246

11. Björ O, Bråbäck L (2003) A retrospective population based trend analysis on hospital admissions for lower respiratory illness among Swedish children from 1987 to 2000. BMC Public Health 3:22

12. Read AW, Gibbins J, Stanley FJ (1996) Hospital admissions for lower respiratory tract illness before the age of two years in western Australia. Paediatr Perinat Epidemiol 10(2):175-185

13. Ramon MA, Gimeno-Santos E, Ferrer J, Balcells E, Rodríguez E, de Batlle J, Gómez FP, Sauleda J, Ferrer A, Barberà JA, Agustí A, Gea J, Rodriguez-Roisin R, Antó JM, Garcia-Aymerich J, PACCOPD Study Group (2014) Hospital admissions and exercise capacity decline in patients with COPD. Eur Respir J 43(4): $1018-1027$

14. De Miguel-Díez J, Jiménez-García R, Hernández-Barrera V, Puente-Maestu L, Rodríguez-Rodríguez P (2013) López de Andrés A, Carrasco-Garrido P. Trends in hospital admissions for acute exacerbation of COPD in Spain from 2006 to 2010. Respir Med 107(5):717-723
15. Mikalsen IB, Skeiseid L, Tveit LM, Engelsvold DH, Øymar K (2015) Decline in admissions for childhood asthma, a 26year period population-based study. Pediatr Allergy Immunol. doi:10.1111/pai. 12372

16. De Miguel-Díez J, Jiménez-García R, Hernández-Barrera V, López de Andrés A, Villa-Asensi JR, Plaza V, Carrasco-Garrido P (2014) National trends in hospital admissions for asthma exacerbations among pediatric and young adult population in Spain (2002-2010). Respir Med 108(7):983-991. doi:10.1016/j.rmed.2014.04.008

17. Gibbison B, Griggs K, Mukherjee M, Sheikh A (2013) Ten years of asthma admissions to adult critical care units in England and Wales. BMJ Open 3(9):e003420

18. Barcala FJ, Viñas JA, Cuadrado LV, Bourdin A, Dobaño JM, Takkouche B (2010) Trends in hospital admissions due to asthma in north-west Spain from 1995 to 2007. Allergol Immunopathol (Madr) 38(5):254-258

19. Kinnula VL, Vasankari T, Kontula E, Sovijarvi A, Saynajakangas O, Pietinalho A (2011) The 10-year COPD Programme in Finland: effects on quality of diagnosis, smoking, prevalence, hospital admissions and mortality. Prim Care Respir J 20(2):178-183

20. Rowe BH, Villa-Roel C, Abu-Laban RB, Stenstrom R, Mackey D, Stiell IG, Campbell S, Young B (2010) Admissions to Canadian hospitals for acute asthma: a prospective, multicentre study. Can Respir J 17(1):25-30

21. Wilson DH, Tucker G, Frith P, Appleton S, Ruffin RE, Adams RJ (2007) Trends in hospital admissions and mortality from asthma and chronic obstructive pulmonary disease in Australia, 19932003. Med J Aust 186(8):408-411

22. Engelsvold DH, Oymar K (2003) Hospital admissions for childhood asthma in Rogaland, Norway, from 1984 to 2000. Acta Paediatr 92(5):610-616

23. Kao CC, See LC, Yan DC, Ou LS, Huang JL (2001) Time trends and seasonal variations in hospital admissions for childhood asthma in Taiwan from 1990 to 1998. Asian Pac J Allergy Immunol 19(2): 63-68

24. Crott R, Pouplier I, Roch I, Chen YC, Closon MC (2014) Pneumonia and influenza, and respiratory and circulatory hospital admissions in Belgium: a retrospective database study. Arch Public Health 72(1):33

25. Froes F, Diniz A, Mesquita M, Serrado M, Nunes B (2013) Hospital admissions of adults with community-acquired pneumonia in Portugal between 2000 and 2009. Eur Respir J 41(5):1141-1146

26. Trotter CL, Stuart JM, George R, Miller E (2008) Increasing hospital admissions for pneumonia. England Emerg Infect Dis 14(5): 727-733

27. Monge V, González A (2001) Hospital admissions for pneumonia in Spain. Infection 29(1):3-6

28. Djuretic T, Ryan MJ, Miller E, Fairley CK, Goldblatt D (1998) Hospital admissions in children due to pneumococcal pneumonia in England. J Infect 37(1):54-58

29. Kmietowicz Z (2015) Smoking ban cuts admissions for child respiratory infections. BMJ 350:h2913

30. Humair JP, Garin N, Gerstel E, Carballo S, Carballo D, Keller PF, Guessous I (2014) Acute respiratory and cardiovascular admissions after a public smoking ban in Geneva, Switzerland. PLoS One 9(3):e90417

31. Millett C, Lee JT, Laverty AA, Glantz SA, Majeed A (2013) Hospital admissions for childhood asthma after smoke-free legislation in England. Pediatrics 131(2):e495-e501

32. Grijalva CG, Nuorti JP, Arbogast PG, Martin SW, Edwards KM, Griffin MR (2007) Decline in pneumonia admissions after routine 
childhood immunisation with pneumococcal conjugate vaccine in the USA: a time-series analysis. Lancet 369(9568):1179-1186

33. Puig-Barberà J, Márquez-Calderón S, Masoliver-Fores A, LloriaPaes F, Ortega-Dicha A, Gil-Martín M, Calero-Martínez MJ (1997) Reduction in hospital admissions for pneumonia in noninstitutionalised elderly people as a result of influenza vaccination: a case-control study in Spain. J Epidemiol Community Health 51(5):526-530

34. Herrett E, Shah AD, Boggon R, Denaxas S, Smeeth L, van Staa T, Timmis A, Hemingway H (2013) Completeness and diagnostic validity of recording acute myocardial infarction events in primary care, hospital care, disease registry, and national mortality records: cohort study. BMJ 346:f2350

35. Sanfilippo FM, Hobbs MS, Knuiman MW, Ridout SC, Bradshaw PJ, Finn JC, Rankin JM, Sprivulis PC, Hung J, Sanfilippo FM, Hobbs MS, Knuiman MW, Ridout SC, Bradshaw PJ, Finn JC, Rankin JM, Sprivulis PC, Hung J (2011) Can we monitor heart attack in the troponin era? Evidence from a population-based cohort study. BMC Cardiovasc Disord 11:35 